\title{
STACEE-32: Design, Performance, and Preliminary Results
}

\author{
Rene A. Ong* \\ ${ }^{*}$ Enrico Fermi Institute, The University of Chicago, Chicago IL, 60637
}

\begin{abstract}
The Solar Tower Atmospheric Cherenkov Effect Experiment (STACEE) is designed to detect astrophysical sources of $\gamma$-rays at energies between 25 and $500 \mathrm{GeV}$. STACEE uses large solar mirrors (heliostats) to collect the atmospheric Cherenkov radiation produced in $\gamma$ ray air showers. The use of a large mirror collection area will allow STACEE to probe $\gamma$-ray sources at energies above the reach of the Compton Gamma Ray Observatory (CGRO), but below the reach of conventional Cherenkov telescopes. During the 1998-99 observing season, a portion of STACEE using 32 heliostats was installed at the National Solar Thermal Test Facility (NSTTF) of Sandia National Laboratories (Albuquerque, NM). This initial configuration (STACEE-32) observed a number of astronomical sources, including the Crab and several active galactic nuclei (AGN). Here we report on these observations. We highlight the experimental configuration and the preliminary results from the Crab data. The full STACEE experiment using 64 heliostats will be commissioned in 2000.
\end{abstract}

\section{SCIENTIFIC MOTIVATION}

A revolution in high energy gamma-ray astrophysics began ten years ago with the launch of the EGRET detector aboard the CGRO and with the high significance detection of sources at $\mathrm{TeV}$ energies by ground-based telescopes. EGRET has discovered over 150 sources of $\gamma$-radiation at energies up to $20 \mathrm{GeV}$ [1]. At the same time, improvements in ground-based instruments using the atmospheric Cherenkov technique have resulted in the detection of at least seven point sources at energies above $250 \mathrm{GeV}$ [2]. We now know that very high energy $\gamma$-rays are copiously produced by some of the most powerful astrophysical objects in the Universe, including pulsars, supernova remnants (SNRs) and AGN.

Of the many sources detected by EGRET, most are not yet seen by the ground experiments. For example, more than sixty AGN at a variety of redshifts are seen by EGRET, but only a few nearby sources have been detected from the ground. These results imply that the spectra of AGN cut off at energies between 20 and $250 \mathrm{GeV}$. The fact that only nearby AGN are seen at very high energies suggests that the $\gamma$-rays are absorbed or attenuated on their journey to Earth. High energy $\gamma$-rays interact with photons at infrared (IR) energies via the pair-production process. The level of the intergalactic IR background is not well known, but measurements of absorption features of AGN should 
provide constraints on its flux and spectral shape [3]. In turn, these constraints could give us valuable information about the epoch of galaxy formation and about the composition of dark matter in the Universe [4]. Absorption features might also place important constraints on $\gamma$-ray emission mechanisms for AGN.

For these reasons, and others, the energy range between 20 and $250 \mathrm{GeV}$ is expected to yield a wealth of scientific discovery. To date, however, it remains terra incognita. Exploring the energy window between 20 and $250 \mathrm{GeV}$ is the primary scientific goal for STACEE. A similar experiment, CELESTE, is starting operations at a site in France [5].

\section{STACEE-32 DESIGN}

\section{Concept}

When high energy $\gamma$-rays enter the Earth's atmosphere, they interact and produce extensive air showers of highly relativistic charged particles. These charged particles emit Cherenkov radiation which is beamed to the ground. A pancake of Cherenkov photons approximately $250 \mathrm{~m}$ in diameter strikes the ground within a very short period of time ( 10 ns).

The total amount of Cherenkov light generated in an extensive air shower is directly proportional to the energy of the primary $\gamma$-ray. As one goes down in energy, Cherenkov photon density decreases. Thus, the energy thresholds of atmospheric Cherenkov telescopes are limited by their total mirror collection area. Larger mirrors yield lower energy thresholds.

It has been recognized for some time that existing fields of solar mirrors (heliostats) represent an extremely promising resource for achieving lower energy $\gamma$-ray thresholds. Starting in early 1994, our group developed this idea and made the first detection of atmospheric Cherenkov radiation at a heliostat facility [6]. We also constructed a fullscale prototype detector which we operated at the heliostat field of the NSTTF [7]. Based on the success of our development work, we formulated the design of STACEE (Solar Tower Atmospheric Cherenkov Effect Experiment). The experimental concept is relatively simple. Atmospheric Cherenkov light created in a $\gamma$-ray air shower is reflected by heliostat mirrors to a secondary mirror on a central tower. In turn, this secondary mirror focuses the light onto cameras employing photomultiplier tubes (PMTs). Fast timing circuitry and coincidence multiplicity logic are used to trigger the experiment.

\section{STACEE-32 Optics}

The heliostat layout for the initial STACEE-32 configuration is shown in Figure 1. We used two clumps of heliostats with 16 heliostats in each clump. The Cherenkov photons from each clump were mapped onto a separate $2 \mathrm{~m}$ diameter secondary mirror on the central tower and subsequently onto a separate PMT camera. The secondary mirrors consist of seven hexagonal facets. The facets are made of ground and polished 
float glass that is aluminized on the front surface. The facets are held in position by an aluminum framework so that the overall secondary mirror has a focal length of $2 \mathrm{~m}$. A metal support structure supports the mirror assembly and provides rails for the movable camera.

The camera holds an array of PMT cans. The cans are positioned so that the image from a single heliostat is centered on the entrance aperture of a can and so each can points to the center of the secondary mirror. The can holds a light concentrator coupled to the PMT via a pliable silicone cookie. Each concentrator, called a DTIRC (Dielectric Total Internal Reflection Concentrator), consists of a precisely machined single piece of UV transmitting acrylic. The DTIRCs also define the field of view of each PMT. The PMTs (Photonis XP2282) are fast, bi-alkali tubes that are run at moderate gain $\left(\sim 1.2 \times 10^{5}\right)$.

\section{Electronics and Data Acquisition}

The PMT signals are AC-coupled and amplified (x100) near the cameras. The amplified signals are transmitted via high quality coaxial cable (RG213) to a dedicated electronics room located inside the tower. Copies of the PMT signals are sent to discriminators and to conventional gated ADCs after long $(1 \mu \mathrm{s})$ analog delays. The outputs from the discriminator are used to start conventional multi-hit TDCs and to form the trigger. The trigger requires a time coincidence ( $\sim 20 \mathrm{~ns}$ wide) of the discriminated signals that have been delayed to account for the timing differences existing between the PMTs. The delays are updated every few seconds as a particular source is tracked across the sky. The trigger serves to interrupt the DAQ computer, stop the TDCs, and gate the ADCs. To minimize deadtime, the trigger decision consists of two separate levels: Level 1 , which requires a minimum number of channels within a cluster of eight heliostats to fire in time, and Level 2, which requires a minimum number of clusters to fire.

The data acquisition (DAQ) and online control systems reside on a Silicon Graphics workstation connected to the electronics crates via Ethernet. The online system controls the data-taking and monitors the detector performance. For STACEE-32, the digitizing electronics and the DAQ system were largely built from off-the-shelf components of an older vintage. For STACEE-64, state-of-the-art electronics will be used for both the trigger and digitization functions.

\section{STACEE-32 OPERATIONS AND PERFORMANCE}

\section{Timeline}

The hardware for STACEE-32 was installed during September, 1998, and the experiment was fully operational soon after. Observations with STACEE-32 took place between October, 1998 and April, 1999. In general, the weather was excellent; a large amount of both test and observational data was logged to disk. 


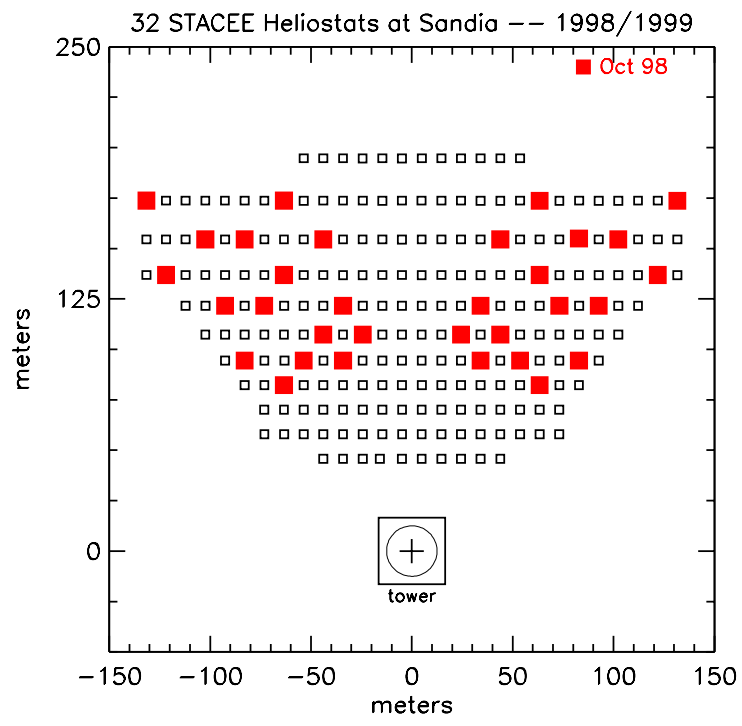

FIGURE 1. Plan view of STACEE-32, an initial version of STACEE that operated during 1998-99. STACEE-32 used 32 heliostats, two secondary mirrors, and 32 photomultiplier tubes. Each heliostat has a mirror area of $37 \mathrm{~m}^{2}$.

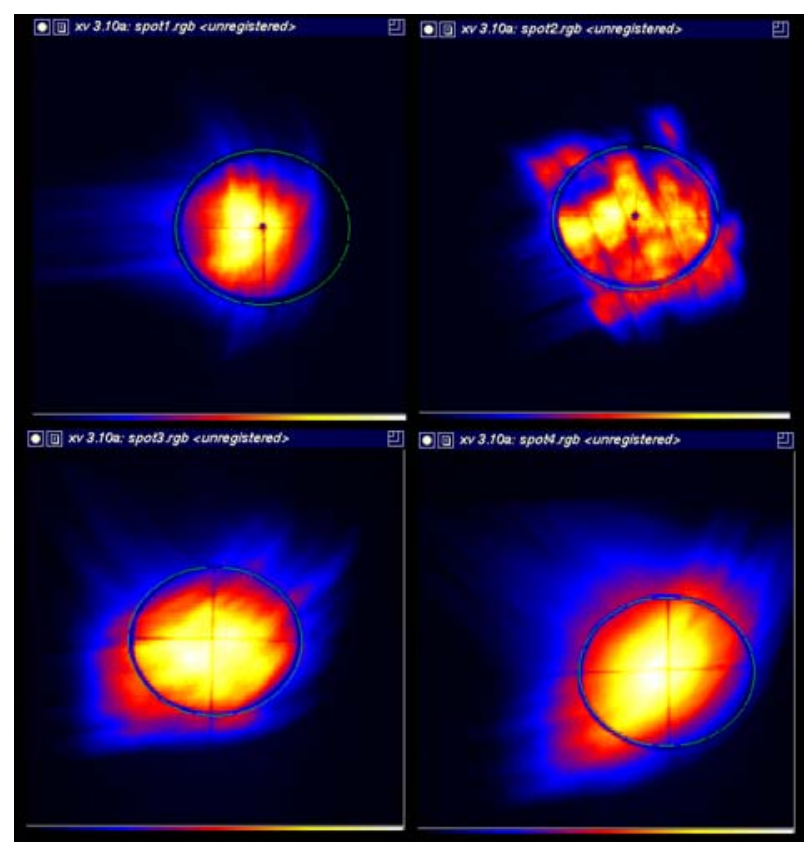

FIGURE 2. Images of the Moon reflected by four individual heliostats into the focal plane of one of the STACEE-32 cameras. The circles indicates the size of the entrance apertures of the PMT cans. 
TABLE 1. Typical operating conditions for STACEE-32. The various items are described in the text.

\begin{tabular}{|lc|}
\hline Item & Typical Values \\
\hline PMT threshold & $6 \mathrm{p} . \mathrm{e}$. \\
PMT rate & $1-10 \mathrm{MHz}$ \\
Trigger condition (L1) & $5 / 8$ \\
L1 rate & $1-20 \mathrm{kHz}$ \\
Trigger condition (L2) & $3 / 4$ \\
L2 rate & $3 \mathrm{~Hz}$ \\
Accidental rate & $<10^{-4} \mathrm{~Hz}$ \\
\hline
\end{tabular}

\section{Heliostats and Alignment}

The heliostats at the NSTTF performed well during the seven months of data taking. Light from the Moon was used to determine the absolute headings for each of the 32 individual heliostats. Moonlight was reflected onto a projection screen on the tower, and the Moon images were recorded by a CCD camera system. Software was developed to allow us to reduce the data from the CCD image immediately and to correct the heliostat alignment. These corrections were made to a precision of one bit on the shaft angle encoders $\left(\sim 0.05^{\circ}\right)$.

Before the observing campaign started, the positions and angles of all the critical STACEE optical components, including mirrors, cameras, and PMTs, were carefully surveyed using a theodolite. The alignment was cross-checked using point sources of light in the field. Moonlight was used to cross-check the optical alignment of the secondary mirrors and the PMT can positions. Figure 2 shows four CCD images of the moonlight projected onto the PMT camera plane. The PMTs were positioned so as to be within $5 \%$ of the optimal light collection efficiency.

\section{Detector Performance}

For the most part, STACEE-32 operated smoothly during the entire observing period. Significant effort was made to characterize the detector performance. These studies were largely made by studying rates at different PMT threshold values and by taking Cherenkov test data at fixed positions in the sky.

Studies on timing properties of each channel were done to verify the correct performance of the trigger and to permit accurate reconstruction of the shower wavefront. Timing offsets and PMT gains for each channel were determined by two different methods: 1) a portable LED flasher which illuminated a single PMT at a time, and 2) a laser calibration system which transmitted a short optical pulse to fibers mounted at the centers of the secondary mirrors.

Typical operating conditions for STACEE-32 are summarized in Table 1. The accidental trigger rate (due to non-Cherenkov events) was calculated from the typical Level 1 rates. Accidentals were negligible at the operating threshold. 
TABLE 2. Raw data (before cuts) taken by STACEE-32, in terms of the numbers of on-source hours.

\begin{tabular}{|lc|}
\hline Source & Data Collected (hours) \\
\hline Crab & 55.3 \\
Markarian 501 & 10.8 \\
Markarian 421 & 6.5 \\
AGN 1219+285 & 7.2 \\
\hline
\end{tabular}

\section{Data Sample}

STACEE-32 observed the Crab Nebula and three AGN. A summary of the amount of data taken on each source is shown in Table 2 . The data were taken in hourly on/off pair intervals (i.e. 28 minutes on-source followed by 28 minutes off-source, or vice-versa). The off-source regions were at the same declination as the on-source regions, but $\pm 7.5^{\circ}$ away in right ascension.

\section{CRAB ANALYSIS}

\section{Pair Cuts}

The total Crab data sample consisted of 133 on/off pairs. We make cuts to remove those pairs that were affected by poor weather, poor or variable atmospheric clarity, or detector malfunctions. We also apply cuts requiring both halves of each pair to be closely matched with regards to their individual tube rates, cluster rates, and other quantities. These cuts ensure that any differences in the numbers of events recorded on-source and those recorded off-source are the result of changes in the Cherenkov rate due to the presence of $\gamma$-rays, as opposed to being due to trigger threshold variations. Overall, the cuts remove 27 pairs, leaving us with 106 pairs for the final data sample.

\section{Event Reconstruction}

The first step of the event reconstruction is to re-impose the trigger condition in software using a $12 \mathrm{~ns}$ coincidence window. The software trigger condition is tighter than that in hardware, but it does not restrict the STACEE field of view. The measured arrival times of the shower at the heliostats are fit to a spherical wave front with a fixed core location (taken as the center of the STACEE-32 array). From the wavefront fit, the shower arrival direction in equatorial coordinates $(\alpha, \delta)$ and a fit quality $\left(\chi^{2}\right)$ are determined. The average reconstructed positions in right ascension and declination agree with the position of the Crab to better than $0.1^{\circ}$ accuracy. From simulations, we expect that $\gamma$-ray showers will have significantly lower values of $\chi^{2}$ than most cosmic ray showers. 
TABLE 3. On-source and off-source totals, the on-off differences, and the significance values for the STACEE-32 Crab data. The various categories are described in the text.

\begin{tabular}{|c|r|r|r|}
\hline & Raw Data & Trigger & Shower Fit \\
\hline ON & 435,738 & 360,695 & 185,047 \\
OFF & 430,301 & 355,346 & 180,530 \\
\hline ON-OFF & 5,437 & 5,349 & 4,517 \\
Signif. & $+5.84 \sigma$ & $+6.32 \sigma$ & $+7.47 \sigma$ \\
\hline
\end{tabular}

\section{DC signal}

In Table 3, we show the total numbers of events for the on-source and off-source portions of each pair. We also show the difference between the on and off portions. The numbers are given for three categories: the raw data, the data after re-imposing the trigger in software, and the data after subsequently requiring the shower fit $\chi^{2}$ to be less than 1.0. The $\chi^{2}$ cut value was chosen from Monte Carlo simulation work. In all three categories there is a statistically significant excess, which improves as successive cuts are applied. Thus, we have confidence that the excess events are due to a signal of $\gamma$-rays from the Crab.

Using the triggered data sample, we have an excess of 5,349 events recorded in a total on-source time of 2,648 minutes. Interpreting the excess events as a steady (DC) $\gamma$-ray signal, STACEE-32 detected $2.02 \pm 0.32 \gamma / \mathrm{min}$ from the Crab. Preliminary Monte Carlo simulations indicate that the peak energy of the detected $\gamma$-rays is near $100 \mathrm{GeV}$. Figure 3 shows that the $\gamma$-ray event rate and the $\gamma$-ray fraction ( $\gamma$-rays/cosmic rays) remained stable over time.

For each on/off pair, we calculate the significance, $(\mathrm{ON}-\mathrm{OFF}) / \sqrt{\mathrm{ON}+\mathrm{OFF}}$, in the excess number of events. The distribution of these 106 significance values is shown in Figure 4. The significance distribution is consistent with a unit-width Gaussian displaced from zero. This distribution gives us confidence that our DC signal is not a statistical artifact or the result of a gross systematic error. After imposing the software trigger, 77 (29) of the pairs have positive (negative) excess.

\section{Crab Pulsar Analysis}

The mechanism that produces the pulsed $\gamma$-ray emission from the Crab is not fully understood. There are two broad classes of models for the pulsed emission: polar cap [8] and outer gap [9]. Determining the pulsed $\gamma$-ray spectrum above $10 \mathrm{GeV}$ could provide a definitive test between the two models. Using the STACEE-32 data, we have made a search for the pulsar component. The arrival times of the on-source events are corrected to the reference frame of the solar barycenter and are folded with the radio pulsar ephemeris obtained by the Jodrell Bank group [10]. Figure 5 shows a histogram where the STACEE-32 events are binned in phase relative to the $33 \mathrm{~ms}$ pulsar period. The phase distribution is consistent with being uniformly distributed (H-test has chance 


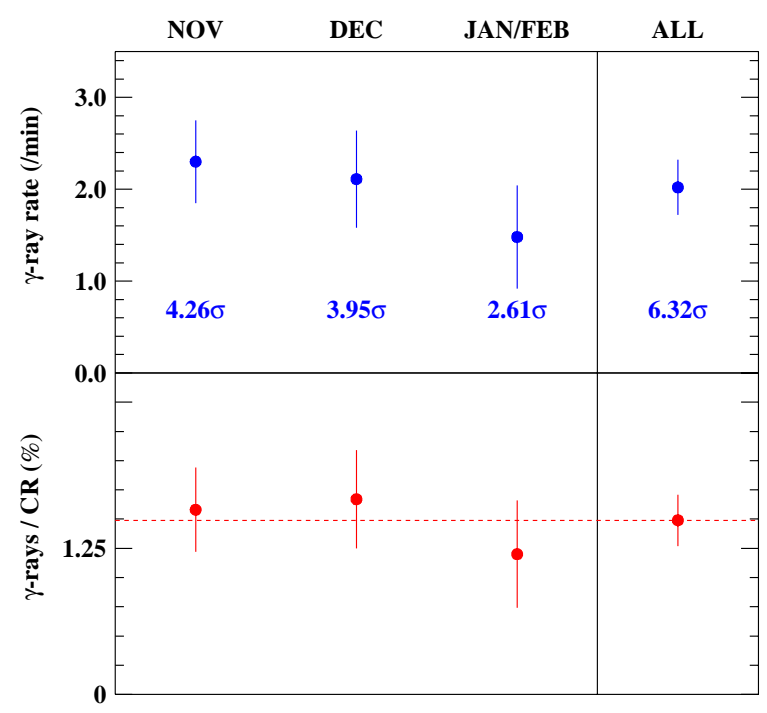

FIGURE 3. Top: STACEE-32 Crab $\gamma$-ray rate for three different observing periods in 1998-99. Bottom: $\gamma$-ray fraction ( $\gamma$-ray rate / cosmic ray rate) for the different periods.

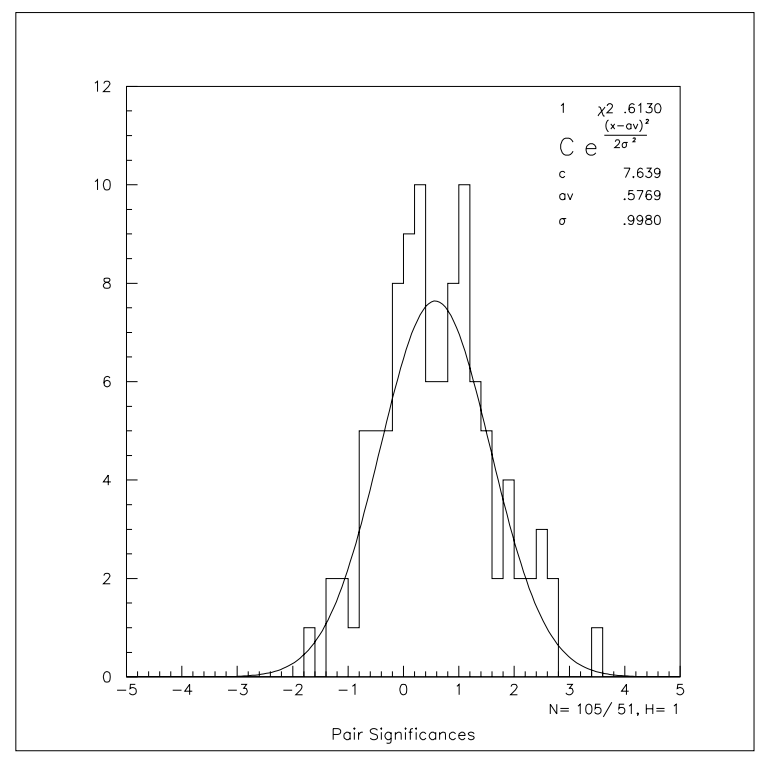

FIGURE 4. On/off pair significances for the STACEE-32 Crab data. The significance are calculated using $(\mathrm{ON}-\mathrm{OFF}) / \sqrt{\mathrm{ON}+\mathrm{OFF}}$. The curve represents a Gaussian fit to the data. 


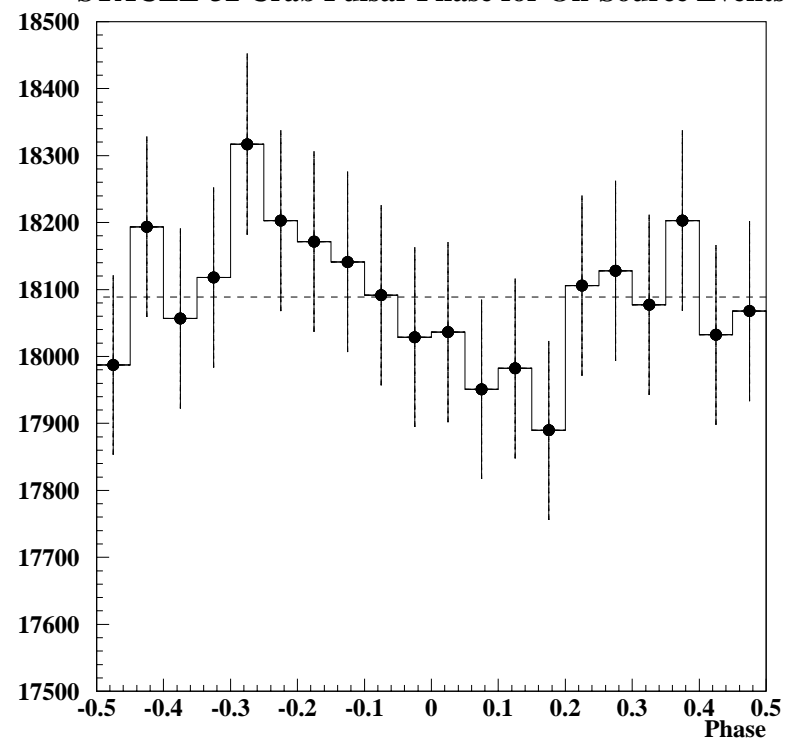

FIGURE 5. Phase diagram for STACEE-32 Crab data. The details of the analysis are given in the text. No strong evidence for pulsed emission from the Crab is seen.

probability for uniformity of 0.24$)$. No strong enhancement is seen in the phase intervals of $(-0.06,0.04)$ and $(0.32,0.43)$ which are those portions of the phase where the $\gamma$-ray pulsed emission peaks [11]. Using these phase portions, we set an upper limit on the pulsed $\gamma$-ray fraction of the emission from the Crab of $<0.025$ (90\% C.L.) at a $\gamma$-ray energy of $\sim 100 \mathrm{GeV}$.

\section{CONCLUSIONS}

STACEE is a new experiment designed to carry out $\gamma$-ray observations in the unopened window between 25 and $250 \mathrm{GeV}$. A portion of the experiment (STACEE-32) was constructed and operated during the 1998-99 season. From 44 hours of on-source observations (along with an equal amount off-source data), STACEE-32 detected a statistically significant excess $(+7 \sigma)$ from the Crab. The observed excess is consistent with being due to $\gamma$-rays at peak energy of $\sim 100 \mathrm{GeV}$. We are in the process of carrying out detailed simulation work in order to accurately estimate the energy response of the detector and in order to determine the $\gamma$-ray flux. No significant evidence for pulsation is seen in our data sample, and we accordingly set an upper limit on the pulsar fraction of the $\gamma$-ray signal. With the addition of more heliostats and new electronics, the full STACEE experiment will be completed in 2000. The full instrument will have significantly improved energy threshold and sensitivity relative to STACEE-32. 


\section{ACKNOWLEDGEMENTS}

STACEE-32 is a collaboration involving the following scientists: D. Bhattacharya, L. Boone, M.C. Chantell, Z. Conner, C.E. Covault, M. Dragovan, D.T. Gregorich, D.S. Hanna, R. Mukherjee, R.A. Ong, S. Oser, K. Ragan, R.A. Scalzo, C.G. Theoret, T.O. Tumer, D.A. Williams, and J.A. Zweerink. We thank the staff of the NSTTF for their excellent support. This work was supported in part by the National Science Foundation, the Natural Sciences and Engineering Research Council, Fonds pour la Formation de Chercheurs et l'Aide a la Recherche, the Research Corporation, and the California Space Institute.

\section{REFERENCES}

1. D. Thompson et al., Astrophys. J. Supp. 101, 259-286 (1995).

2. Rene A. Ong, Physics Reports 305, 93-202 (1998).

3. F.W. Stecker, Astropart. Phys. 11, 83-91 (1999).

4. J.R. Primack et al., Astropart. Phys. 11, 93-102 (1999).

5. D.A. Smith, Proceedings of this Workshop.

6. R.A. Ong et al., Astropart. Phys. 5, 353-365 (1996).

7. M.C. Chantell et al., Nucl. Inst. Meth. A408, 468-485 (1998).

8. See, for example: J.K. Daugherty and A.K. Harding, Astrophys. J. 458, 278-292 (1996).

9. See, for example: J. Chiang and R. Romani, Astrophys. J. 400, 629-637 (1994).

10. Jodrell Bank Crab Pulsar Timing Results Monthly Ephemeris, A.G. Lyne, R.S. Pritchard, M.E. Roberts, April 28, 1999.

11. See for example: P.V. Ramanamurthy et al., Astrophys. J. 450, 791-804 (1995). 